\title{
ON THE CHERNOUS'KO TIME-OPTIMAL PROBLEM FOR THE EQUATION OF HEAT CONDUCTIVITY IN A ROD ${ }^{1}$
}

\author{
Abdulla A. Azamov ${ }^{\dagger}$, Jasurbek A. Bakhramov ${ }^{\dagger \dagger}$, Odiljon S. Akhmedov ${ }^{\dagger \dagger}$ \\ Institute of Mathematics, National University of Uzbekistan named after Mirzo Ulugbek, \\ Durmon yuli st., 29 Tashkent, 100125, Uzbekistan \\ †abdulla.azamov@gmail.com, \\ ††bahramov.jasurbek@gmail.com, ${ }^{\dagger \dagger}$ odiljon.axmedov@gmail.com
}

\begin{abstract}
The time-optimal problem for the controllable equation of heat conductivity in a rod is considered. By means of the Fourier expansion, the problem reduced to a countable system of one-dimensional control systems with a combined constraint joining control parameters in one relation. In order to improve the time of a suboptimal control constructed by F.L. Chernous'ko, a method of grouping coupled terms of the Fourier expansion of a control function is applied, and a synthesis of the improved suboptimal control is obtained in an explicit form.
\end{abstract}

Keywords: Heat equation, Time-optimal problem, Pontryagin maximum principle, Suboptimal control, Synthesis of control.

\section{Introduction}

It is known that a time-optimal problem occupied a very important place in the foundation and development of optimal control theory. Even for simple non-trivial cases, the problem required working-out new approaches and lead after all to Pontryagin's maximum principle [3, 10, 30]. Despite 70 years of development, the solution of concrete non-trivial examples of time-optimal control still needs considerable effort $[2,4,19]$. The problem becomes even more difficult when a control system is described by a partial differential equation [11, 24, 25, 34], particularly, for the heat conductivity equation $[12,22,26,29,35,36]$. In [1], the correctness of parabolic equations for heat propagation is discussed and for that purpose, a parabolic equation with time delay is considered.

Here, the maximum principle can be formally written out as well, but it loses its effectiveness as compared with a finite-dimensional case or on cases when the time interval is fixed $[2,9,18,25$, 32, 33]. Therefore, Chernous'ko suggested [13] another approach based on the Fourier expansion that allowed him to reduce the problem to an infinite system of one-dimensional problems whose control parameters are connected by a condition in the min-max form (see below (1.4)) generating a closed convex control set in a Hilbert space. Unfortunately, to deal with such a constraint is quite difficult (about other kinds of constraints see [17]). In order to overcome this complexity, the mentioned constraint was replaced [13] by an infinite system of separated conditions for scalar control parameters that can be interpreted as if one took Hilbert's brick inscribed into the control set. As a result, this approach made it possible to construct a suboptimal control and to give an explicit upper estimation for an optimal time. In [5], a co-Hilbert's brick inscribed into the control set was considered, and an improved suboptimal control function was constructed. In the present

\footnotetext{
${ }^{1}$ This work was supported by a grant from the Ministry of Innovative Development of the Republic of Uzbekistan (Project No. OT- $\Phi 4-84$ ).
} 
paper, we suggest another way for constructing a suboptimal control function in the case of the heat conductivity equation in a rod.

\section{Preliminaries}

As it was noted above, Chernous'ko considered the time-optimal problem for an evolutional equation

$$
\frac{\partial u(t, x)}{\partial t}=A[u(\cdot, \cdot)](t, x)+v(t, x)
$$

with the initial and boundary conditions

$$
u(0, x)=u^{0}(x), \quad M u(t, s)=u^{*}(t, s),
$$

where $A$ is a uniformly elliptic differential operator, $t \geq 0, x \in D, D$ is a regular domain with Lyapunov boundary $\Gamma, s \in \Gamma$, and $M$ is a boundary operator [13].

The constraint on the control function in problem (1.1), (1.2) is bounded in the norm of the space $L_{\infty}$; i.e. $|v(t, x)| \leq v_{0}$ for almost all $t$ and every $x \in \bar{D}$, where $v_{0}$ is a given positive number [31]. It is known that, for every control function $v(t, x)$, problem (1.1), (1.2) has a unique solution $u(t, x)[14,21,28]$.

If a solution $u(t, x)$ of problem (1.1), (1.2) satisfies the condition $u(T, x) \equiv 0$ at some $T, T \geq 0$, then the corresponding control function $v(t, x)$ is called admissible, and the number $T$ is called the transition time (from the initial state $u_{0}(\cdot)$ into the equilibrium state $u(t, x) \equiv 0$ ). Let $V$ be the class of all admissible controls. Then the quantity $T=T[v(\cdot, \cdot)]$ will be a functional on $V$ at every fixed $u^{0}(x)$ and $u^{*}(t, s)$.

If an admissible control $v_{*}(t, x)$ satisfies the condition $T_{*}=T\left[v_{*}(\cdot, \cdot)\right] \leq T[v(\cdot, \cdot)]$ for all $v(\cdot, \cdot) \in V$, then $v_{*}(\cdot, \cdot)$ is called a time-optimal control, and the value $T_{*}$ is called optimal transition time.

The direct application of the Pontryagin maximum principle to problem (1.1), (1.2) is a very hard task, unlike optimization problems on a finite interval of time (see $[4,8,15]$ ). For example, in [25], only theorems on the existence of optimal control and the bang-bang principle are given, but no specific example of a solution was considered. In monograph [11], the time-optimal problem when a control parameter participates in boundary conditions was considered [11, Ch. 5, Sect. 1] and, instead of the necessary conditions, the method of the $L$-momentum of N.N. Krasovskii [19] was applied [11, Sect. 2]. In the recently published article [20], Butkovsky's approach was applied to the case of a fractional-order diffusion equation. It should be noted that the $L$-momentum method only allows one to simplify to some degree the time-optimal problem and rarely gives an explicit solution. Therefore, the approach suggested by Chernous'ko [13], where the method of expansions on the system of eigenfunctions of the operator $A$ was used, seems to be more effective. That helped to reduce considering problem to the infinite system of one-dimensional control problems:

$$
\dot{y}_{k}=-\lambda_{k} y_{k}+v_{k}, \quad y_{k}(0)=y_{k 0}, \quad k=0,1,2, \ldots .
$$

(About solution of systems of this kind, see [16]).

In terms of system (1.3), the condition $|v(t, s)| \leq v_{0}$ means that a counting system of the control parameters $v_{k}, k=0,1,2, \ldots$, should satisfy the combined constraint

$$
\max _{x \in \bar{D}}\left|\sum_{k=0}^{\infty} \varphi_{k}(x) v_{k}\right| \leq v_{0}
$$

where $\varphi_{k}$ are eigenfunctions of the problem. 
Condition (1.4) defines some closed convex set $L$ in the Hilbert space $l_{2}$, which is difficult to deal with. In this connection, it is natural to try to solve the problem of finding a suboptimal control. (It is essential to note that, if a time interval is fixed, then the method of penalty functions is enough effective for the construction of a suboptimal control. It would be interesting to apply this method for the time-interval problem as well [6].) For this purpose, in [13], constraint (1.4) was replaced by a more rigid system of constraints in the form

$$
\left|v_{k}\right| \leq U_{k}, \quad k=0,1,2, \ldots,
$$

where $\alpha_{k}=\max _{x \in \bar{D}}\left|\varphi_{k}(x)\right|$. Wherein, nonnegative numbers $U_{k}$ should be chosen satisfying the condition $\sum_{k=0}^{\alpha} \alpha_{k} U_{k}=v_{0}$.

Let $T_{* k}$ be an optimal transition time in the problem

$$
\dot{y}_{k}=-\lambda_{k} y_{k}+v_{k}, \quad y_{k}(0)=y_{k}^{0},
$$

such that $y_{k}\left(T_{* k}\right)=0, k=0,1,2 \ldots$ In [13], it is shown that the numbers $U_{k}$ can be chosen so that all $T_{* k}$ coincide: $T_{* k}=\hat{T}$ for some $\hat{T}$. Let $\hat{v}_{k}(t)$ be the sequence of the corresponding optimal controls. Then $T_{*} \leq \hat{T}$ and $\hat{v}_{*}(t, x)=\sum_{k=0}^{\infty} \varphi_{k}(x) v_{* k}(t)$ may serve as the sought suboptimal control.

A new problem arises here: is it possible to use a more exact reduction of the constraint than (1.5)? As mentioned above in [5] it was used Hilbert's co-cube instead of (1.5). Here, we are going to follow another approach based on a special grouping of terms of (1.5). Effectiveness of this approach is tightly related to specific properties of eigenfunctions $\varphi_{k}(\cdot)$, so here it will be demonstrated for the operator $A=\partial^{2} / \partial x^{2}$ connected with the process of the heat conductivity in a rod.

\section{A method of grouping terms of the Fourier expansion}

Consider the following concretization of problem (1.1), (1.2):

$$
\left\{\begin{array}{l}
\frac{\partial u}{\partial t}=\frac{\partial^{2} u}{\partial x^{2}}+v(t, x), \quad|v(t, x)| \leq v_{0}, \quad t \geq 0, \quad 0 \leq x \leq \pi, \\
u(0, x)=u^{0}(x), \quad u(t, 0)=0, \quad u(t, \pi)=0 .
\end{array}\right.
$$

The system of eigenfunctions $\varphi_{k}(t)=\sin k x, k=1,2, \ldots$, of the operator $\partial^{2} / \partial x^{2}$ forms a complete orthogonal basis of the space $L_{2}[0, \pi][21,28]$.

Let $u(t, x)=\sum_{k=1}^{\infty} y_{k} \sin k x$ and $v(t, x)=\sum_{k=1}^{\infty} v_{k} \sin k x$ be the Fourier expansions on the basis $\{\sin k x\}$. Then the restriction (1.4) takes the form

$$
\max _{0 \leq x \leq \pi}\left|\sum_{k=1}^{\infty} v_{k} \sin k x\right| \leq v_{0} .
$$

Let us consider a more rigid restriction

$$
\max _{0 \leq x \leq \pi} \sum_{k \in Q}\left|v_{k} \sin k x+v_{3 k} \sin 3 k x\right| \leq v_{0}
$$

instead of (2.2), thereby replacing the optimal control problem with a suboptimal control problem.

System (1.6) takes the form

$$
\dot{y}_{k}=-k^{2} y_{k}+v_{k}, \quad k \in Z^{+} .
$$


Let $Q$ be the set of all positive integers having the form $3^{2 p} q$, where $p=0,1,2, \ldots$, and $q$ is relatively prime with 3 . It is obvious that the set of all positive integers $Z^{+}$is the union of the two disjoint sets $Q$ and $3 Q$. Then (2.4) can be rewritten in the form

$$
\dot{y}_{k}=-k^{2} y_{k}+v_{k}, \quad \dot{y}_{3 k}=-9 k^{2} y_{3 k}+v_{3 k}, \quad k \in Q .
$$

After the substitutions

$$
y_{k}=\frac{\mu_{k}}{k^{2}} x^{1}, \quad y_{3 k}=\frac{\mu_{k}}{k^{2}} x^{2}, \quad t=\frac{1}{k^{2}} \tau, \quad v_{k}=\mu_{k} w^{1}, \quad v_{3 k}=\mu_{k} w^{2},
$$

all systems (2.5) will be reformulated to the following two-dimensional control system:

$$
\dot{x}^{1}=-x^{1}+w^{1}, \quad \dot{x}^{2}=-9 x^{2}+w^{2} .
$$

Now, following the Chernous'ko way, we replace (2.3) by the even more rigid restriction

$$
\max _{0 \leq x \leq \pi}\left|w_{k}^{1} \sin k x+w_{k}^{2} \sin 3 k x\right| \leq 1, \quad k \in Q
$$

that implies (2.3) if $\sum_{k \in Q} \mu_{k}=v_{0}$. Thus, we have reduced the infinite dimensional control problem to the two-dimensional problem.

\section{Solution of the auxiliary time-optimal problem on the plane}

Let $P_{k}$ denote the set of all pairs $\left(w_{k}^{1}, w_{k}^{2}\right)$ for which (2.7) holds. Setting

$$
P=\left\{w=\left(w^{1}, w^{2}\right) \in R^{2}: \max _{0 \leq t \leq \pi}\left|w^{1} \sin t+w^{2} \sin 3 t\right| \leq 1\right\},
$$

we have $P_{k}=\mu_{k} P$. As a result, the considered problem of constructing a suboptimal control reduces to the concrete problem of time-optimal control for the following two-dimensional system:

$$
\dot{x}^{1}=-x^{1}+w^{1}, \quad \dot{x}^{2}=-9 x^{2}+w^{2}, \quad\left(w^{1}, w^{2}\right) \in P .
$$

Obviously, $P$ is a convex and compact set with non-empty interior (i.e., a convex body). Since $P$ is symmetric with respect to the origin, we may restrict ourselves to considering only the case $w^{1} \geq 0$. It is more convenient to set $\sin t=y$. Then, by the formula

$$
\sin 3 t=3 \sin t-4 \sin ^{3} t,
$$

we get

$$
P=\left\{w=\left(w^{1}, w^{2}\right) \in R^{2}: \max _{0 \leq y \leq 1}\left|\left(w^{1}+3 w^{2}\right) y-4 w^{2} y^{3}\right| \leq 1\right\} .
$$

Just this transformation lay on the base of the separation $Z^{+}=Q \cup 3 Q$.

After elementary calculations, we find that the part of the boundary of the set $P$ lying in the half-plane $w^{1} \geq 0$ is given by the formula

$$
w^{1}=\left\{\begin{array}{llr}
w^{2}+1 & \text { if } & -1 \leq w^{2}<0.125, \\
3\left(\sqrt[3]{w^{2}}-w^{2}\right) & \text { if } & 0.125 \leq w^{2} \leq 1,
\end{array}\right.
$$

while the other part is found by central symmetry (see Fig. 1).

Let us recall that, in the auxiliary problem (3.1), a unique optimal time-control function exists at each initial point $\left(x_{0}^{1}, x_{0}^{2}\right)[7,23,27]$. The existence follows from the property $O \in \operatorname{Int} P$. The 


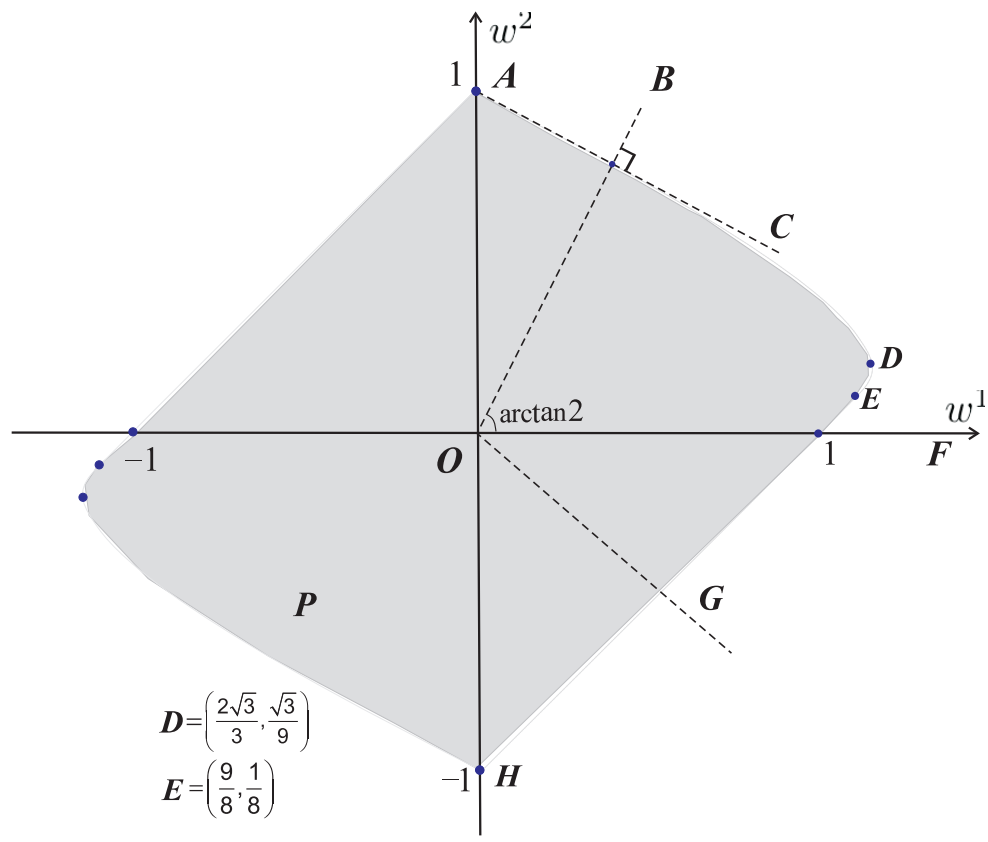

Figure 1. The straight ray $A C$ is tangent at the point $A$ to the curve $A D$, which is a part of the boundary of $P$.

uniqueness is a consequence of the following feature of $P$ : the vector $(1,1)$, which is orthogonal to the segment $A C$, is not an eigenvector of the matrix of system (3.1). Therefore, the optimal control problem (2.5) coincides with the extremal controls of Pontryagin's maximum principle [23, 30].

To calculate the latter, we prefer to use the "backward motion" principle. Let $T\left(x_{0}^{1}, x_{0}^{2}\right)$ be a transition time for the initial point $\left(x_{0}^{1}, x_{0}^{2}\right)$ in the system (3.1). If we set $\tau=T\left(x_{0}^{1}, x_{0}^{2}\right)-t$, then extremals of Pontryagin's maximum principle are defined by the system

$$
\left\{\begin{array}{c}
\frac{d x^{1}}{d \tau}=x^{1}-\bar{w}^{1}, \quad \frac{d x^{2}}{d \tau}=9 x^{2}-\bar{w}^{2}, \quad \frac{d \psi_{1}}{d \tau}=-\psi_{1}, \quad \frac{d \psi_{2}}{d \tau}=-9 \psi_{2} . \\
x(0)=y(0)=0, \quad \psi_{1}(0)=\cos s, \quad \psi_{2}(0)=\sin s, \quad-\pi \leq s \leq \pi
\end{array}\right.
$$

Since $\psi_{1}(\tau, s)=e^{-\tau} \cos s$ and $\psi_{2}(\tau, s)=e^{-9 \tau} \sin s$, an extremal control $\bar{w}(\tau, s)$ should be found by the Pontryagin's maximum principle, i.e., from the equation

$$
\bar{w}^{1}(\tau, s) e^{-\tau} \cos s+\bar{w}^{2}(\tau, s) e^{-9 \tau} \sin s=\max _{w \in P}\left[w^{1} e^{-\tau} \cos s+w^{2} e^{-9 \tau} \sin s\right] .
$$

Equation (3.3) leads to the following construction of the extremal controls.

If $\psi(\tau, s)$ lies in the open angle $A O B$, then obviously $\bar{w}(\tau, s)=(0,1)$. Note that, if $s=\pi / 2$, then $\psi_{1}(\tau, s) \equiv 0$. Therefore, $\bar{w}(\tau, \pi / 2)=(0,1)$. Similarly, if $s=0$, then $\psi_{2}(\tau, s) \equiv 0$; thus, $\bar{w}(\tau, 0)=(2 \sqrt{3} / 3, \sqrt{3} / 9)$.

Consider now the dynamics of $\psi(\tau, s)$. In the case $0<s<\pi / 2$, the vector $\psi(\tau, s)$ lies in the quarter $\psi_{1}>0, \psi_{2}>0$ and turns clockwise. Moreover, its direction tends to the axis of abscissas $O E$ as $\tau \rightarrow+\infty$. (Similarly, if $-\pi / 2<s<0$, then $\psi(\tau, s)$ lies in the quarter $\psi_{1}>0$, $\psi_{2}<0$ and turns counterclockwise with the same limit direction.)

Thus, the extremal control has the following structure: if $0<s \leq \arctan 2$ (see Fig. 1), then $\psi(\tau, s)$ lies in the angle $B O D$ for all $\tau(\tau \geq 0)$, and, hence, $\bar{w}(\tau, s)$ is a point of the arc $A D$ such that its projection to the direction $\psi(\tau, s)$ is maximal (the analytical expression for $\bar{w}(\tau, s)$ is given in Table 1). 
Abdulla A. Azamov, Jasurbek A. Bakhramov

Table 1. The analytical expression for $\bar{w}^{1}(\tau, s)$ and $\bar{w}^{2}(\tau, s)$.

\begin{tabular}{|c|c|cc|}
\hline & $\bar{w}^{1}(\tau, s)$ & \multicolumn{2}{|c|}{$\bar{w}^{2}(\tau, s)$} \\
\hline$-\frac{\pi}{2}<s \leq-\frac{\pi}{4}$ & $0 \quad$ if $0 \leq \tau \leq \tau_{* *}$, & $-1 \quad$ if $0 \leq \tau \leq \tau_{* *}$, \\
$M$ & if $\tau_{* *} \leq \tau$ \\
\hline$-\pi / 4<s<0$ & $3\left(2-e^{-8 \tau} \tan s\right) M \quad$ if $\tau_{* *} \leq \tau$ & & $M$ \\
\hline$s=0$ & $3\left(2-e^{-8 \tau} \tan s\right) M$ & & $\sqrt{3} / 9$ \\
\hline $0<s \leq \arctan 2$ & $2 \sqrt{3} / 3$ & & $M$ \\
\hline $\arctan 2<s<\frac{\pi}{2}$ & $3\left(2-e^{-8 \tau} \tan s\right) M$ & 1 & if $0 \leq \tau \leq \tau_{*}$, \\
\hline$s=\pi / 2$ & 0 & if $0 \leq \tau \leq \tau_{*}$, & if $\tau_{*} \leq \tau$ \\
\hline
\end{tabular}

Table 2. The analytical expression for $x^{1}(\tau, s)$ and $x^{2}(\tau, s)$.

\begin{tabular}{|c|c|c|}
\hline & $x^{1}(\tau, s)$ & $x^{2}(\tau, s)$ \\
\hline$-\frac{\pi}{2}<s \leq-\frac{\pi}{4}$ & $\frac{3 e^{\tau}}{4 \tan s} \int_{m}^{n} \frac{1-p^{2}}{p^{2} q^{7}} d p$ if $\quad \tau_{* *} \leq \tau$ & $\frac{e^{9 \tau}}{4 \tan s} \int_{m}^{n} q d p \quad$ if $\tau_{* *} \leq \tau$ \\
\hline$-\pi / 4<s<0$ & $\frac{3 e^{\tau}}{4 \tan s} \int_{m}^{n} \frac{1-p^{2}}{p^{2} q^{7}} d p$ & $\frac{e^{9 \tau}}{4 \tan s} \int_{m}^{n} q d p$ \\
\hline$s=0$ & $2 \sqrt{3}\left(1-e^{\tau}\right) / 3$ & $\sqrt{3}\left(1-e^{9 \tau}\right) / 81$ \\
\hline $0<s \leq \arctan 2$ & $\frac{3 e^{\tau}}{4 \tan s} \int_{m}^{n} \frac{1-p^{2}}{p^{2} q^{7}} d p$ & $\frac{e^{9 \tau}}{4 \tan s} \int_{m}^{n} q d p$ \\
\hline $\arctan 2<s<\frac{\pi}{2}$ & $\frac{3 e^{\tau}}{4 \tan s} \int_{m}^{n} \frac{1-p^{2}}{p^{2} q^{7}} d p$ if $\tau_{*} \leq \tau$ & $\frac{e^{9 \tau}}{4 \tan s} \int_{m}^{n} q d p \quad$ if $\quad \tau_{*} \leq \tau$ \\
\hline$s=\pi / 2$ & 0 & $\left(1-e^{9 \tau}\right) / 9$ \\
\hline
\end{tabular}

Further, in the case $\arctan 2<s<\pi / 2$, we have $\bar{w}(\tau, s)=(0,1)$ on the interval $\left[0, \tau_{*}\right)$, where $\tau_{*}=-1 / 8 \cdot \log (2 \cot s)$. At the time $\tau=\tau_{*}$, the vector $\psi(\tau, s)$ becomes orthogonal to the right side tangent to the curve $\partial P$ at the point $(0,1)$ and it occurs "switching" of the extremal control from the value $(0,1)$ to a continuous mode. Namely, $\bar{w}(\tau, s)$ begins sliding along the arc $A C$ (see Table 1) and tends to the point $C$ as $\tau \rightarrow+\infty$.

Similarly, if $(-\pi / 2<s<-\pi / 4)$, then $\bar{w}(\tau, s)=(0,-1)$ at $0 \leq \tau<\tau_{* *}$, where $\tau_{* *}=$ $-1 / 8 \cdot \log (-\cot s)$ and $w(\tau, s)$ is a switching time. On the interval $\left(\tau_{* *},+\infty\right), \bar{w}(\tau, s)$ slides along the arc $E D$ tending to the point $D$.

The entire synthesis of the extremal control is given in Table 1. Due to the central symmetry, the values of $s$ are considered only on the range $-\pi / 2<s \leq \pi / 2$ and the following notation is used:

$$
\begin{gathered}
M=\left(3-e^{-8 \tau} \tan s\right)^{-3 / 2}, \quad m=\left(3 e^{8 \tau} \cot s-1\right)^{-1 / 2}, \quad n=(3 \cot s-1)^{-1 / 2}, \\
p=\left(3-e^{-8 \tau} \tan s\right)^{-1 / 2}, \quad q=\left(\left(3-p^{-2}\right) \cot s\right)^{1 / 8} .
\end{gathered}
$$


Now, extremal trajectories can be easily calculated by (3.2). The corresponding formulas are gathered in Table 2. They are illustrated in Fig. 2.

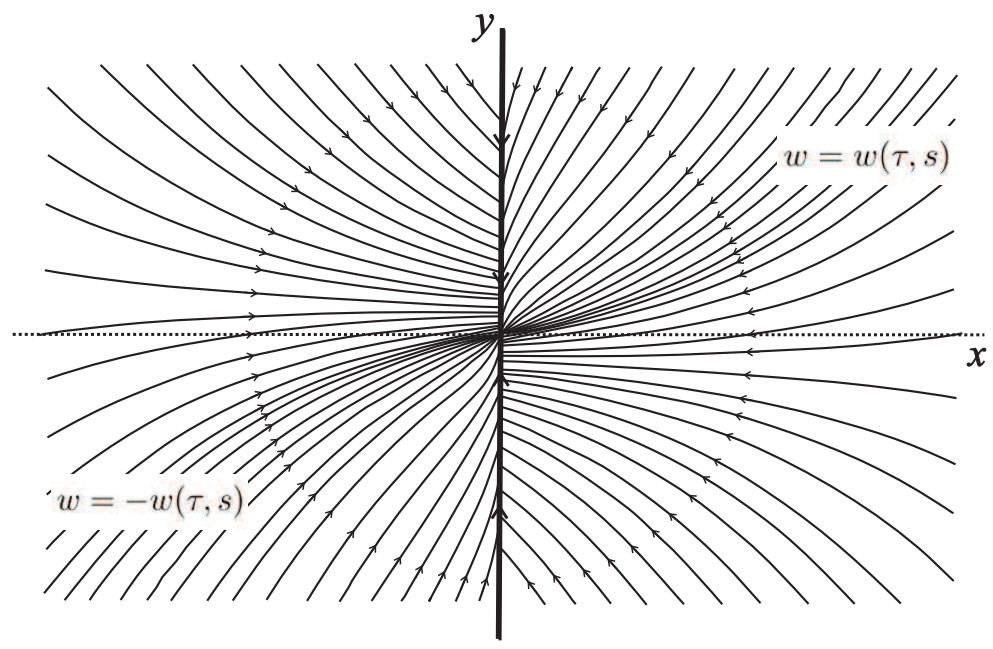

Figure 2. The extremal trajectories.

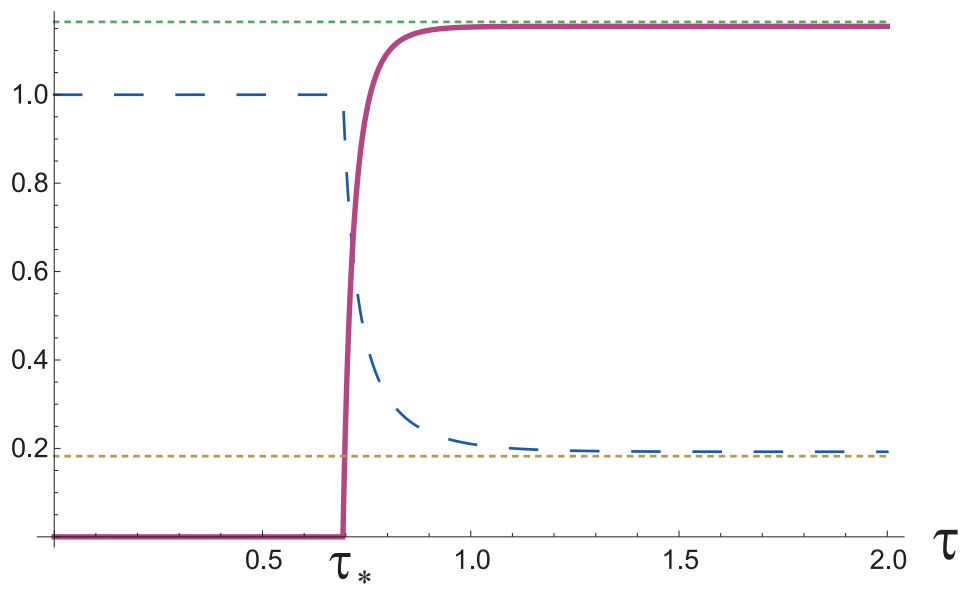

Figure 3. The graphs of the functions $\bar{w}^{1}(\tau, s)$ (the continuous line) and $\bar{w}^{2}(\tau, s)$ (the dashed line) for $\arctan 2<s<\pi / 2$.

\section{Construction of a suboptimal control in the initial problem}

Let us now derive the solution of problem (2.1), (2.3), (2.5) basing one of reduced problems.

Extremals $x(t, s), y(t, s)$ cover $R^{2}$. Therefore, for every $\left(x_{0}^{1}, x_{0}^{2}\right) \in R^{2} \backslash(0,0)$, there exists a pair $\left(\tau_{0}, s_{0}\right)$ such that $x^{1}\left(\tau_{0}, s_{0}\right)=x_{0}^{1}, x^{2}\left(\tau_{0}, s_{0}\right)=x_{0}^{2}$. Further, in the system (3.1), for every fixed $\left(x_{0}^{1}, x_{0}^{2}\right) \neq 0$, an optimal control is unique, which implies the uniqueness of the value $\tau_{0}$ (while corresponding values of $s_{0}$ may be not unique, but one can choose any of them).

Then $T\left(x_{0}^{1}, x_{0}^{2}\right)=\tau_{0}$ is the transition time and

$$
\bar{v}^{1}(t)=v^{1}\left(\tau_{0}-t, s_{0}\right), \quad \bar{v}^{2}(t)=v^{2}\left(\tau_{0}-t, s_{0}\right)
$$




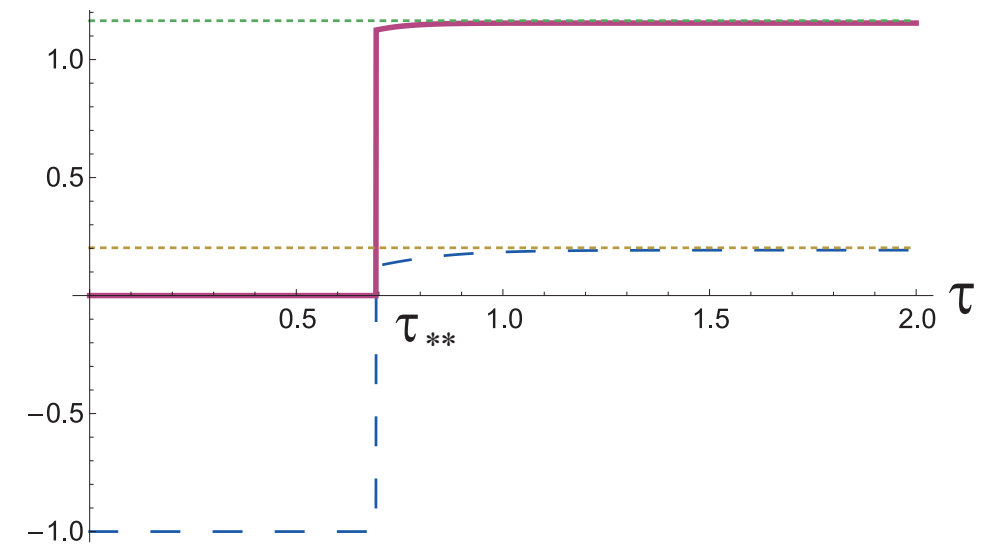

Figure 4. The graphs of the functions $\bar{w}^{1}(\tau, s)$ (the continuous line) and $\bar{w}^{2}(\tau, s)$ (the dashed line) for $-\pi / 2<s<\arctan 2$.

is the suboptimal control for (2.1).

Let us now consider system (2.5). For an initial point $\left(y_{k}^{0}, y_{3 k}^{0}\right)$, the corresponding trajectory $\left(y_{k}(t), y_{3 k}(t)\right)$ satisfies the condition

$$
y_{k}\left(T_{k}\right)=y_{3 k}\left(T_{k}\right)=0
$$

where

$$
T_{k}\left(\mu_{k}\right)=\frac{1}{k^{2}}\left(\frac{k^{2}}{\mu_{k}} y_{k}^{0}, \frac{k^{2}}{\mu_{k}} y_{3 k}^{0}\right) .
$$

The constructed synthesis implies that $T_{k}$ is monotonically decreasing in $\mu_{k}$, and it is easy to see that $T_{k} \rightarrow 0$ as $\mu_{k} \rightarrow+\infty$ and $T_{k} \rightarrow \infty$ as $\mu_{k} \rightarrow 0$. Therefore, for every $k$, there exists a unique value $\mu_{k}^{*}$ such that $T_{k}\left(\mu_{k}^{*}\right)$ is the same for all $k$. Moreover, $\mu_{k}^{*}$ can be chosen satisfying the condition $\sum \mu_{k}^{*}=v_{0}$. One can easily see that

$$
\frac{\alpha}{k^{2}} \leq \mu_{k}^{*} \leq \frac{\beta}{k^{2}}
$$

for some positive $\alpha$ and $\beta$.

Finally, we consider the initial problem (2.1), (2.3), (2.5). Let $u_{0}(x)=\sum_{k=1}^{\infty} u_{k}^{0} \sin x$ be the Fourier expansion of the initial function $u_{0}(x)$. Taking $\left(y_{k}^{0}, y_{3 k}^{0}\right), k \in Q$, as an initial point for system (2.6), we find

$$
\bar{w}_{k}^{0}(t)=\frac{1}{\mu_{k}} \bar{v}_{k}^{1}(t), \quad \bar{w}_{3 k}^{0}(t)=\frac{1}{\mu_{k}} \bar{v}_{k}^{2}(t), \quad k \in Q .
$$

Thus, the following statement holds.

Theorem 1. The function

$$
\bar{v}(t, x)=\sum_{k=1}^{\infty} \bar{w}_{k}^{0}(t) \sin k x
$$

is a suboptimal control in problem (2.1) for the initial state $u_{0}(x)$.

\section{Conclusion}

The paper is devoted to the time-optimal problem for the process of heat conductivity in a rod when the control parameter is the intensity of external heat sources. A suboptimal control is 
constructed by the combination of the Chernous'ko approach with the method of grouping terms of the Fourier expansion.

This method may be applied to the time-optimal control problem for other systems given in an evolutionary form.

The following question naturally arises: how effective is the method of grouping? First of all, let us bring general considerations. The set of all admissible controls in the initial problem (1.1)-(1.2) can be identified with the subset

$$
U_{\text {Initial }}=\left\{u \in l_{2}\left|\sup _{0 \leq x \leq \pi}\right| \sum_{k=0}^{\infty} u_{k} \sin k x \mid \leq v_{0}\right\} .
$$

As noted in Section 1, Chernous'ko restricted the set of controls using

$$
U_{C h}=\left\{u \in l_{2}|| u_{k} \mid \leq U_{k}, \quad k=1,2,3, \ldots\right\}
$$

where $U_{k}$ is a sequence chosen from the condition $\sum U_{k} \leq v_{0}$ and guaranteeing the equality $u(t, x) \equiv 0$ for some $T=T_{C h}>0$.

The considerations in this paper are based on the set

$$
U_{g r}=\left\{u \in l_{2}\left|\max _{0 \leq x \leq \pi}\right| u_{k} \sin k x+u_{3 k x} \sin 3 k x \mid \leq U_{k}\right\}
$$

taken as a region of admissible controls.

One can easily see that

$$
U_{g r} \subset U_{C h} \subset U_{\text {Initial }} \text {. }
$$

These relations imply $T_{2} \leq T_{1} \leq T_{0}$ for optimal and suboptimal times of transition respectively.

If one takes an initial point of the form $\left(0,0, \ldots, x_{m}^{0}, 0, \ldots, 0\right)$, i.e., in terms of the initial problem (1.1)-(1.2), $\varphi(x)=\left(0,0, \ldots, x_{m}^{0} \sin m x, 0, \ldots, 0\right)$, then, obviously, $U_{\text {Initial }}=U_{C h}=U_{g r}$ and, thus, $T_{2}=T_{1}=T_{0}$. But if an initial point is taken in the form $\left(0,0, \ldots, 0, x_{k}^{0}, 0, \ldots, 0, x_{3 k}^{0}, 0, \ldots\right)$, then $U_{I n}=U_{g r}$ while $U_{I n} \supset U_{C h}$ and, thus, $T_{2}=T_{0}<T_{1}$. Table 3 contains values for specific cases.

Table 3

\begin{tabular}{|c|c|c|}
\hline Initial point & $T_{1}$ & $T_{0}=T_{2}$ \\
\hline$(1,0,1,0,0,0, \ldots)$ & 0.7 & 0.64 \\
\hline$(1,0,2,0,0,0, \ldots)$ & 0.67 & 0.61 \\
\hline$(1,0,-1,0,0,0, \ldots)$ & 0.72 & 0.65 \\
\hline$(1,0,-2,0,0,0, \ldots)$ & 0.69 & 0.62 \\
\hline
\end{tabular}

Obviously, $x_{k}^{0} \neq 0$ at least for three values of the index $k$ when $T_{2}<T_{0}<T_{1}$.

The final note is that the method of grouping can be applied only if there some algebraic relations between the eigenfunctions of the operator $A$.

\section{REFERENCES}

1. Ahmed N. U. Optimal control of infinite dimensional systems governed by integro differential equations. In: Differential Equations: Dynamical Systems, and Control Science. Eds. K.D. Elworthy, W.N. Everitt, E.B. Lee. Ser. Lect. Notes Pure Appl. Math., 1994. Vol. 152. P. 383-402.

2. Alekseev V.M., Tikhomirov V.M., Fomin S. V. Optimal'noe upravlenie [Optimal Control]. Moscow: Nauka, 2005. 384 p. (in Russian) 
3. Arutyunov A. V., Karamzin D. Y., Pereira F. M. The maximum principle for optimal control problems with state constraints by R.V. Gamkrelidze: Revisited. J. Optim. Theory Appl., 2011. Vol. 149, No. 3. P. 474-493. DOI: 10.1007/s10957-011-9807-5

4. Arutyunov A.V., Vinter R. B. A simple 'Finite Approximations' proof of the Pontryagin maximum principle under reduced differentiability hypotheses. Set-Valued Anal., 2004. Vol. 12, No. 1-2. P. 5-24. DOI: 10.1023/B:SVAN.0000023406.16145.a8

5. Azamov A. A., Ruzibayev M. R. The time-optimal problem for evolutionary partial differential equations. J. Appl. Math. Mech., 2013. Vol. 77, No. 2. P. 220-224. DOI: 10.1016/j.jappmathmech.2013.07.013

6. Barbu V. Analysis and Control of Nonlinear Infinite Dimensional Systems. Ser. Math. Sci. Eng., Vol. 190. Boston: Academic Press, MA, 1993. 475 p.

7. Blagodatskikh V.I. Vvedenie $v$ optimal'noe upravlenie (linejnaya teoriya). [Introduction to Optimal Control (Linear Theory)]. Moscow: Vysshaya Shkola, 2001. 239 p. (in Russian)

8. Bongini F., Fornasier M., Rossi F., Solombrino F. Mean-field Pontryagin maximum principle. J. Optim. Theory Appl., 2017. Vol. 175, No. 1. P. 1-38. DOI: 10.1007/s10957-017-1149-5

9. Bonnet B., Rossi F. A Pontryagin Maximum Principle in Wasserstein Spaces for Constrained Optimal Control Problems. 2018. arXiv: 1810.13117v2 [math.OC]

10. Bryson A. E. Optimal Control - 1950 to 1985. IEEE Control Systems, 1996. Vol. 16, No. 3. P. $26-33$. DOI: $10.1109 / 37.506395$

11. Butkovskiy A. G. Metody upravleniya sistemami s raspredelennymi parametrami [Control Methods of the Systems with Distributed Parameters]. Moscow: Nauka, 1965. 474 p. (in Russian)

12. Carthel C., Glowinski R., Lions J.L. On exact and approximate boundary controllability for the heat equation: A numerical approach. J. Optim. Theory Appl., 1994. Vol. 82, No. 3. P. $429-484$. DOI: $10.1007 /$ BF02192213

13. Chernous'ko F.L. Bounded controls in distributed-parameter systems. J. Appl. Math. Mech., 1992. Vol. 56, No. 5. P. 707-723. DOI: 10.1016/0021-8928(92)90057-F

14. Evans L. C. Partial Differential Equations. 2-nd ed. Ser. Grad. Stud. Math., Vol. 19. Providence, Rhode Island: Amer. Math. Soc., 2010. 749 p.

15. Gong W., Hinze M., Zhou Z. A priori error analysis for finite element approximation of parabolic optimal control problems with pointwise control. SIAM J. Control Optim., 2014. Vol. 52, No. 1. P. $97-119$. DOI: $10.1137 / 110840133$

16. Ibragimov G., Risman M.H., Azamov A. A. Existence and uniqueness of the solution for an infinite system of differential equations. J. Karya Asli Lorekan Ahli Matematik, 2008. Vol. 1, No. 2. P. 9-14.

17. Ibragimov G. I. Optimal pursuit time for a differential game in the Hilbert space $l_{2}$. Science Asia, 2013. Vol. 39S, No. 1. P. 25-30. DOI: 10.2306/scienceasia1513-1874.2013.39S.025

18. Ji G., Martin C. Optimal boundary control of the heat equation with target function at terminal time. Appl. Math. Comput., 2002. Vol. 127, No. 2-3. P. 335-345. DOI: 10.1016/S0096-3003(01)00011-X

19. Krasovskii N. N. Teoriya upravleniya dvizheniem [Theory of Control of Motion]. Moscow: Nauka, 1968. 476 p. (in Russian)

20. Kubyshkin V.A., Postnov S.S. Time-optimal boundary control for systems defined by a fractional order diffusion equation. Autom. Remote Control, 2018. Vol. 79, No. 5. P. 884-896. DOI: 10.1134/S0005117918050090

21. Ladyzhenskaya O.A. The boundary value problems of mathematical physics. Ser. Appl. Math. Sci., vol. 49. New York: Springer-Verlag, 1985. 322 p. DOI: 10.1007/978-1-4757-4317-3

22. Laykekhman D., Vexler B. Optimal a priori error estimates of parabolic optimal control problems with pointwise control. SIAM J. Numer. Anal., 2013. Vol. 51, No. 5. P. 2797-2821. DOI: 10.1137/120885772

23. Lee E. B., Markus L. Foundation of Optimal Control Theory. Malabar (FL): Krieger Pub Co, 1986. $586 \mathrm{p}$.

24. Lee M. J., Park J. Y. Pontryagin's maximum principle for optimal control of a non-well-posed parabolic differential equation involving a state constraint. ANZIAM J., 2004. Vol. 46, No. 2. P. 171-184. DOI: $10.1017 /$ S1446181100013778

25. Lions J. L. Optimal Control of Systems Governed by Partial Differential Equations. Ser. Grundlehren Math. Wiss., Vol. 170. Berlin, Heidelberg: Springer-Verlag, 1971. 440 p.

26. Lü Q., Wang G. On the existence of time optimal controls with constraints of the rectangular type for heat equations. SIAM J. Control Optim., 2011. Vol. 49, No. 3. P. 1124-1149. DOI: 10.1137/10081277X 
27. Magaril-Ilyaev G. G., Tikhomirov V. M. Convex Analysis: Theory and Applications. Ser. Transl. Math. Monogr. Providence, USA: Amer. Math. Soc., 2003. 183 p.

28. Mizohata S. The Theory of Partial Differential Equations. Cambridge: University Press, 1979.490 p.

29. Pan L. P., Yong J. Optimal control for quasilinear retarded parabolic systems. ANZIAM J., 2001. Vol. 42, No. 4. P. 532-551. DOI: 10.1017/S1446181100012268

30. Pontryagin L.S., Boltyanskii V. G., Gamkrelidze R. V., Mishchenko E. F. The Mathematical Theory of Optimal Processes. NY, London: John Wiley \& Sons, 1962. 320 p.

31. Raymond J.P., Zidani H. Pontryagin's Principle for state-constrained control problems governed by parabolic equations with unbounded controls. SIAM J. Control Optim., 1998. Vol. 36, No. 6. P. 18531879. DOI: 10.1137/S0363012996302470

32. Raymond J.P., Zidani H. Pontryagin's principle for time-optimal problems. J. Optim. Theory Appl., 1999. Vol. 101, No. 2. P. 375-402. DOI: 10.1023/A:1021793611520

33. Ross I. M. A Primer on Pontryagin's Principle in Optimal Control. Collegiate Publishers, 2009.109 p.

34. Serag H. M. Distributed control for cooperative systems involving parabolic operators with an infinite number of variables. Pure Math. Appl., 2004. Vol. 15, No. 4. P. 439-451.

35. Tsachev T. An optimal control problem for the heat equation. Mathematica Balkanica, 1984. Vol. 3. P. 296-310. URL: http://www.math.bas.bg/infres/MathBalk/MB-03/MB-03-296-310.pdf

36. Zhang Y. On a kind of time optimal control problem of the heat equation. Adv. Difference Equ., 2018. Vol. 2018. Art. no. 117. P. 1-10. DOI: 10.1186/s13662-018-1577-z 